DRAFT VERSION SEPTEMBER 10, 2018

Preprint typeset using LTEX style emulateapj v. 08/17/12

\title{
PROPERTIES AND MODELING OF UNRESOLVED FINE STRUCTURE LOOPS OBSERVED IN THE SOLAR TRANSITION REGION BY IRIS
}

\author{
DAVID H. BROOKS ${ }^{1,3}$, JeFFREY W. REEP ${ }^{2,4}$, AND HARRY P. WARREN ${ }^{2}$ \\ ${ }^{1}$ College of Science, George Mason University, 4400 University Drive, Fairfax, VA 22030, USA and \\ ${ }^{2}$ Space Science Division, Naval Research Laboratory, Washington, DC 20375, USA \\ Draft version September 10, 2018
}

\begin{abstract}
Recent observations from the Interface Region Imaging Spectrograph (IRIS) have discovered a new class of numerous low-lying dynamic loop structures, and it has been argued that they are the long-postulated unresolved fine structures (UFS) that dominate the emission of the solar transition region. In this letter, we combine IRIS measurements of the properties of a sample of 108 UFS (intensities, lengths, widths, lifetimes) with 1-D non-equilibrium ionization simulations using the HYDRAD hydrodynamic model to examine whether the UFS are now truly spatially resolved in the sense of being individual structures rather than composed of multiple magnetic threads. We find that a simulation of an impulsively heated single strand can reproduce most of the observed properties suggesting that the UFS may be resolved, and the distribution of UFS widths implies that they are structured on a spatial scale of $133 \mathrm{~km}$ on average. Spatial scales of a few hundred $\mathrm{km}$ appear to be typical for a range of chromospheric and coronal structures, and we conjecture that this could be an important clue to the coronal heating process.
\end{abstract}

Subject headings: Sun: chromosphere-Sun: corona-Sun: UV radiation

\section{INTRODUCTION}

From Skylab data analysis, Feldman (1983) identified several discrepancies between observations of the emission from the transition region, which he defined as the solar plasma in the $0.02-1.0 \mathrm{MK}$ temperature range, and the predictions of classical models of the interface connecting the chromosphere and corona. First, the emission above the solar limb comes from more extended heights than the thin transition layer expected from one continuous structure. Second, there is a large discrepancy between the observed emission at $0.25 \mathrm{MK}$ and the predictions of theoretical models (Athay 1982). Feldman (1983) argued that these observations, together with other evidence based on off-limb line width measurements and electron densities in different solar regions, suggest that most of the transition region emission originates in unresolved fine structures (UFS) that are magnetically isolated from the chromosphere and corona. Subsequently, Feldman (1987), Feldman (1998), and Feldman et al. (2001) presented further evidence for this view. In particular, Feldman (1998) showed that the plasma composition enhancement at transition region temperatures is different than that at coronal temperatures, supporting the idea that the UFS are disconnected from the corona, and have relatively shorter lifetimes than coronal structures.

This suggestion led to theoretical developments such as the "cool loop" model of Antiochos \& Noci (1986). The growing awareness of the temporal variability of the solar atmosphere, however, led others to question the existence of UFS based on classical models of the transition region modified to incorporate dynamic effects (Wikstøl et al. 1998). Without instruments with sufficient spatial resolution to settle the debate, however, these diverging views were not reconciled.

The launch of the Interface Region Imaging Spectrograph (IRIS, De Pontieu et al. 2014), however, has provided a new

\footnotetext{
${ }^{3}$ Current address: Hinode Team, ISAS/JAXA, 3-1-1 Yoshinodai, Chuoku, Sagamihara, Kanagawa 252-5210, Japan.

${ }^{4}$ National Research Council Postdoctoral Fellow.
}

opportunity to observe the transition region with high spatial resolution and high cadence. Using IRIS, Hansteen et al. (2014) discovered numerous rapidly-varying low-lying loops at transition region temperatures and argued that these are the UFS, whose existence was predicted by Feldman (1983). Hansteen et al. (2014) found that these loops are highly dynamic; often showing red and/or blue shifted velocities of $80 \mathrm{~km} \mathrm{~s}^{-1}$ or more. They have half lengths of $2-6 \mathrm{Mm}$, reach heights of $1-4.5 \mathrm{Mm}$ and are about three times brighter on average than spicules. They light up in segments and are shortlived, though systems of several may persist for tens of minutes. Since they have only recently been detected little else is known about their properties, and it has not been demonstrated that they are the dominant contributors to the transition region emission. That could instead come from spicules (De Pontieu et al. 2011), and their relative contributions remain to be quantified. Throughout this work, however, we have followed the argument of Hansteen et al. (2014) and refer to the UV loops studied here as UFS.

IRIS has the sensitivity and resolution to detect the UFS, but the question arises as to whether that sensitivity and resolution is sufficient to spatially resolve them as single monolithic structures, or whether they are bundles of subresolution magnetic strands that remain spatially unresolved. This point was raised by Hansteen et al. (2014), who noted that UFS that appear as monolithic structures in IRIS observations show substructure in their numerical simulations, and is a question of recent interest because the answer defines the requirements for future solar instrumentation that we hope can determine the true properties of solar atmospheric structures. It also guides our theoretical thinking, because many chromospheric and coronal heating mechanisms are expected to release energy on very small spatial scales ( 10's of meters). Physical models of observations of coronal loops from Hinode, $\mathrm{SDO}$, and $\mathrm{Hi}$ - $\mathrm{C}$, however, suggest that we are already close to resolving them with current instruments, and that they have spatial scales of a few hundred $\mathrm{km}$ (Brooks et al. 2012, 2013). A few hundred km seems 

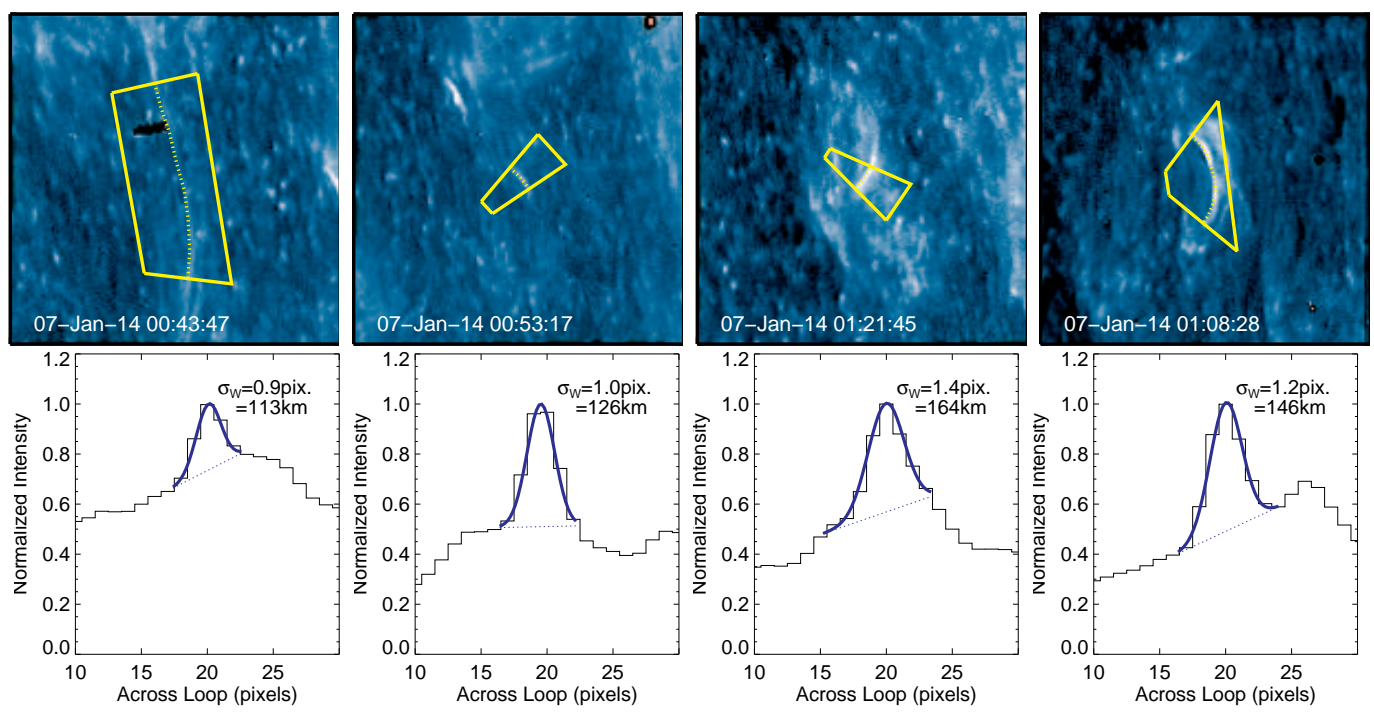

FIG. 1.- Example UFS segments. Top row: IRIS slit-jaw images (SJI) with the loop segments highlighted in yellow. These images have been sharpened with a Gaussian filter. Bottom row: cross-field normalized intensity profiles (solid histogram) with Gaussian fits (blue solid line) and backgrounds (blue dotted line) overlaid. The Gaussian width in IRIS pixels and $\mathrm{km}$ is shown in the legend. The interpolated data have been resampled to show the instrument pixel scale.

to be a common scale for many solar atmospheric structures from Type II spicules (Pereira et al. 2012) to "coronal rain" condensations (Antolin \& Rouppe van der Voort 2012), flaring and post-flare loops (Cirtain et al. 2013; ; Jing et al. 2016), and even prominence threads (Okamoto et al. 2007). So, if correct, the challenge becomes how to convert the small scale energy release into this typical preferred size.

Here we present new measurements of the spatial scales of the UFS. We also use the observed lifetimes, half-lengths, and peak intensities as input to numerical hydrodynamic simulations of the cooling times of the UFS. By comparing the model with the IRIS data, we conclude that the observed lifetimes are consistent with a simulation of a single thread, suggesting that they may be spatially resolved.

\section{OBSERVATIONS}

De Pontieu et al. (2014) describe IRIS in detail. Here we only use Si IV $1400 \AA$ slit-jaw images. The data were obtained between 2013 December 9 and 2014 January 7 using the IRIS observing ID 3800259453, which takes 8s exposures of a $120^{\prime \prime}$ by $129^{\prime \prime}$ field-of-view and was run near the solar limb where it is relatively easier to identify UFS. The spatial pixel size is $0.167^{\prime \prime}$. The data have been processed, calibrated, and coaligned to level-2 and were obtained from the search facility at Lockheed Martin.

We examined $\sim 5$ hours of observations and visually identified 108 UFS. Many of them brighten only partially, or the brightenings move along the loop, or the UFS themselves move rapidly and change dimensions. So there may be some selection bias towards UFS that are visually prominent and relatively easier to isolate from surrounding features. During our study, however, our analysis techniques were developed to better handle structures that are moving or changing shape.

To measure the UFS intensities and widths we used the same procedure as in our earlier work on coronal loop properties (Warren et al. 2008; Brooks et al. 2012, 2013). The method is based on the work of Aschwanden et al. (2008), and extracts the cross-UFS intensity profile by interpolating along the structure within a selected segment, straightening it, and averaging the intensities along the UFS axis. A firstorder polynomial is then fit to the background between two selected positions in the intensity profile, and a Gaussian fit is made to the background subtracted profile. The UFS intensity and width $\left(\sigma_{W}\right)$ are then the area and width of the Gaussian, respectively. Examples are shown in Figure 1

The same segment, UFS axis, and background definitions are then used to extract the intensities for every image in the data cube in order to prepare a light curve for measuring the lifetimes. As mentioned, there is a problem here if the UFS are moving or changing dimensions, because the selected axis may not be co-located with the structure at all times. This issue also sometimes occurs for UFS that are long-lived because the IRIS coalignment can be affected by spacecraft pointing drift while observing the limb. The automatic coalignment procedure and post-processing by cross-correlation do not always fully correct for this because they tend to lock on to the slit portion and fiducial marks in the slit-jaw images. To mitigate this problem, we allow for dynamic movement of the selected background positions in the cross-UFS intensity profile. Since these movements are always smaller than the range of the perpendicular segment, the averaged profile includes the UFS even if it has moved, and the dynamic movement of the background positions ensures that the extracted intensity and width are correct and reflect what is observed in the images.

Example UFS light curves are shown in Figure 2. We fit a polynomial background and Gaussian function to the light curve of each UFS, and take the full width at half maximum (FWHM) as the lifetime $\left(\sigma_{L}\right)$. The peak intensity $\left(I_{P}\right)$ of the Gaussian is also recorded for each UFS. Note that the degree of the background polynomial used in the fit is dependent on how prominent the UFS light curve is above the background. Also, sometimes the UFS brightens and fades but remains visible for some time. In these cases we fit multiple Gaussians to the light curve and sum the FWHMs to get the lifetime. The fourth panel in Figure 2] shows an example of such a case. Fi- 

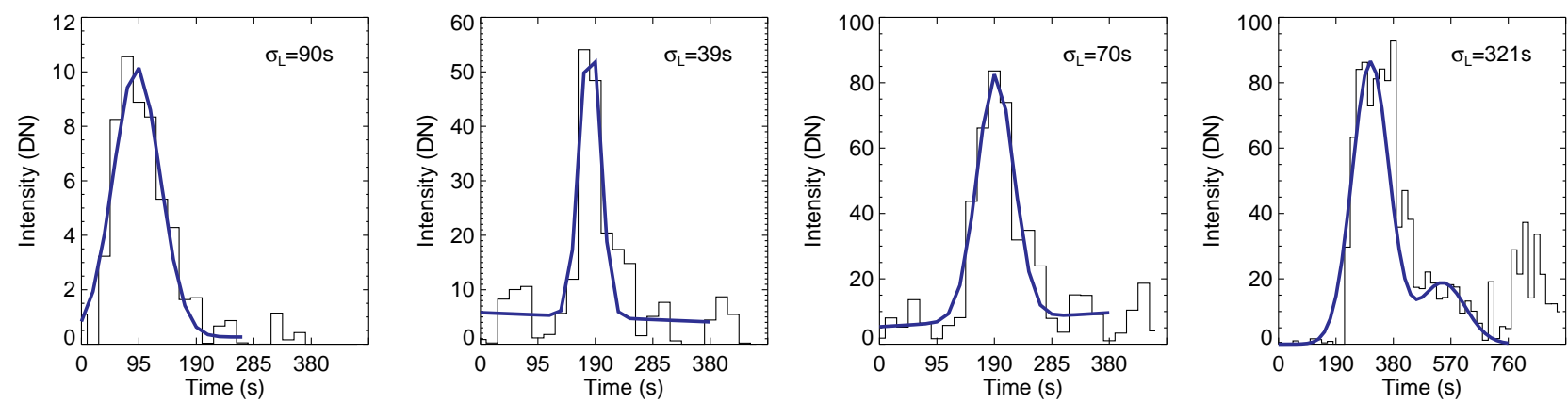

FIG. 2. - Light curves for the UFS in Figure 1 We show the extracted intensities (histogram) with Gaussian fits (blue line) overlaid. The FWHM of the Gaussian fit for each case is given in the legend.
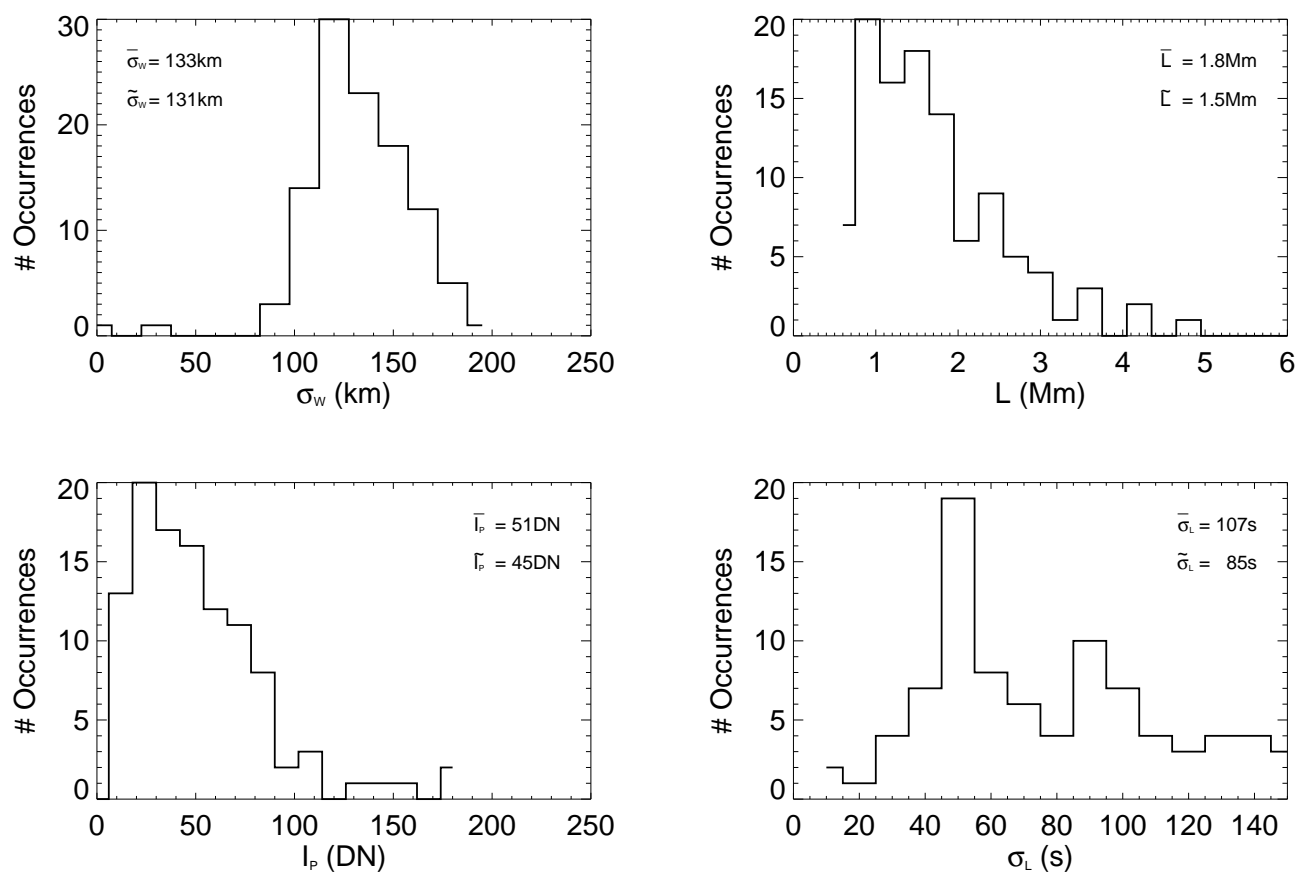

FIG. 3.- Histograms displaying our analysis results for the sample of UFS. Top left: Distribution of cross-field (Gaussian) widths, $\sigma_{W}$, in km. Top right: Distribution of half-lengths, $L$, in Mm. Bottom left: Distribution of peak intensities, $\mathrm{I}_{p}$, in DN. Bottom right: Distribution of UFS FWHM lifetimes, $\sigma_{L}$, in s. The mean and median values are indicated in the legend.

nally, we also measure the UFS half-length $(L)$ by extracting the cross-UFS intensity profile averaged along the half-length of the structure, straightening it, and measuring the straightened length.

The results of our analysis are shown as histograms in Figure 3. The UFS widths fall in the range $35-192 \mathrm{~km}$ with an average of $133 \mathrm{~km}$; the UFS half-lengths fall in the range 0.6$9.5 \mathrm{Mm}$ with an average of $1.8 \mathrm{Mm}$; the UFS peak intensities fall in the range 9-182 DN with an average of $51 \mathrm{DN}$; and the UFS lifetimes fall in the range 5-323 s with an average of $107 \mathrm{~s}$. These peak intensities and lifetimes are consistent with the results of Hansteen et al. (2014). Our half-lengths are towards, and smaller than, the lower range of their values, but this can likely be explained by the different analysis methods.
Hansteen et al. (2014) do not quote any width measurements.

We note that the exact nature of these distributions is not clear from our limited sample size. It could be that they are actually power-law distributions, in which case the means would not be defined.

\section{SIMULATIONS}

We have run simulations with the HYDrodynamics and RADiation code (HYDRAD; Bradshaw \& Mason 2003; Bradshaw \& Cargill 2013), which solves the hydrodynamic equations appropriate to a two-fluid plasma confined to an isolated, one-dimensional magnetic flux tube. We treat radiative losses with a full calculation, using emissivities from CHIANTI v.8 (Dere et al. 1997; Del Zanna et al. 2015), as- 

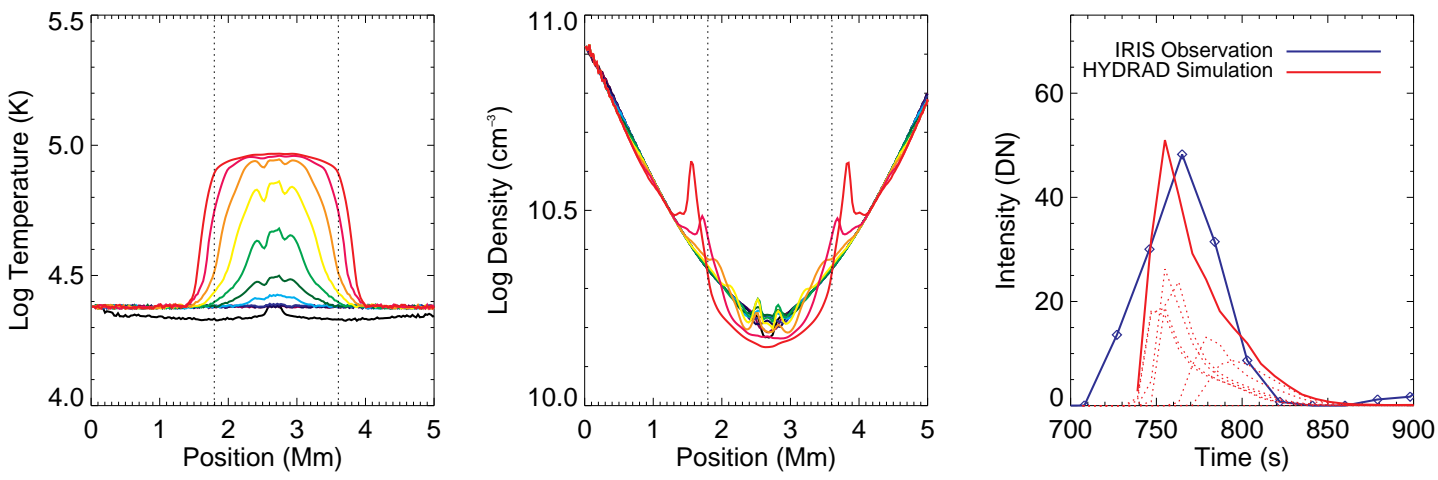

FIG. 4.- Simulation of the properties of one of the UFS in our sample. This UFS has a half-length of $0.9 \mathrm{Mm}$, a peak intensity of $47.1 \mathrm{DN}$ and a lifetime of $50.7 \mathrm{~s}$. Left panel: The distribution of temperature along the loop during the simulation. Middle panel: the distribution of density. The temperature and density distributions are plotted every $5 \mathrm{~s}$ going from blue to red. The vertical dotted lines show that a loop of the required half-length $(0.9 \mathrm{Mm})$ is formed. Right panel: Comparison between the simulated (red) and observed (blue) intensities for the same UFS. The total intensity of the half-loop is shown with the solid red line. The intensities of a subset of the individual pixels are shown with dotted red lines. Only half of the individual pixels are shown for clarity.
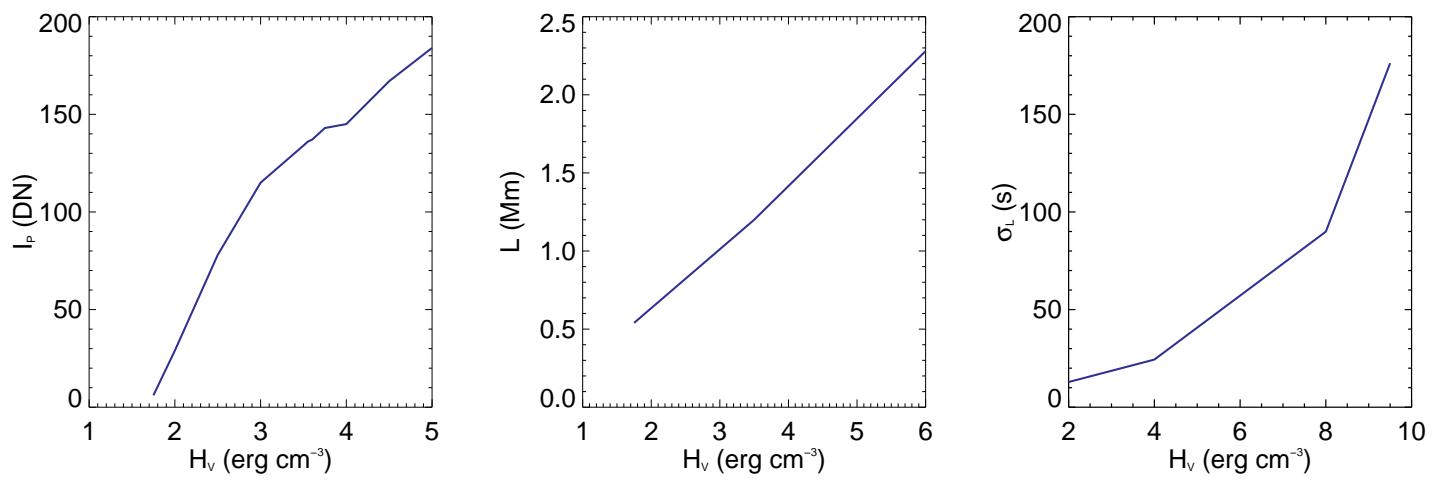

FIG. 5.- Summary plots of the simulated UFS peak intensity, $\mathrm{I}_{p}$, half-length, $L$, and lifetime, $\sigma_{L}$, as a function of the volumetric heating, $\mathrm{H}_{V}$.

suming photospheric abundances (Asplund et al. 2005), and allow for non-equilibrium ionization of hydrogen and silicon. We calculate the Si IV emission line following the methodology of Bradshaw \& Klimchuk (2011), using the IRIS instrumental response from iris_get_response in SSW.

We assume that the loop begins with a flat temperature profile of $24 \mathrm{kK}$, i.e. that the loop has not been heated to coronal temperatures initially and that there is no background heating term. We then heat the loop uniformly, with a triangular temporal profile, for a given duration and maximum heating rate. As the loop evolves, we calculate the Si IV emission and test for consistency with the observed values of intensity, lifetime, and loop length.

Figure 4 shows a simulation that reproduces the properties of one of the UFS in the sample. This UFS has a half-length of $0.9 \mathrm{Mm}$, a peak intensity of $47.1 \mathrm{DN}$ and a lifetime of $50.7 \mathrm{~s}$. The volumetric heating rate in the simulation is $6 \times 10^{-2}$ erg $\mathrm{cm}^{-3} \mathrm{~s}^{-1}$ and the heating duration is $100 \mathrm{~s}$. The temperature increases to $\log \mathrm{T}=4.96$ and the density decreases to $\log \mathrm{N}_{e}=$ 10.15. This forms a loop of the required average half-length $(0.9 \mathrm{Mm})$ emitting very close to the temperature of the peak of the Si IV $1393.755 \AA$ contribution function $(\log \mathrm{T}=4.9)$, which is the strongest Si IV line in the SJI passband.

Figure 4 also compares the totaled intensity over the loop as a function of time for the simulation with the light-curve of the same UFS. The simulated Si IV 1393.755 $\AA$ emission has been scaled down by a factor of 16 , in this example only, to match the peak intensity because the line-of-sight depth is an unknown parameter in the simulation. More importantly, the observed intensity profile is modeled quite well and a Gaussian fit to the simulated intensity profile gives a lifetime of $43 \mathrm{~s}$, which is within $20 \%$ of the observed value.

We also plot the contributions to the total intensity from several individual pixels in the simulation (dotted lines). Interestingly, these are offset in time and have different peak brightnesses. This reproduces another aspect of the observations that was noted by Hansteen et al. (2014) and discussed earlier: that the UFS light up in segments with different intensities at different times.

We found that the model can also reproduce most of the properties of the entire sample of UFS if we modify the volumetric heating rate or heating duration. Figure 5 illustrates this by showing the peak intensity, half-length, and lifetime of the simulated UFS as a function of, $\mathrm{H}_{V}$, the volumetric heating (product of rate and duration). The range of values of $\mathrm{H}_{V}$ shown produce peak intensities of 6.1-184 DN, halflengths of $0.5-2.3 \mathrm{Mm}$, and lifetimes of 13-176 s. These simulated ranges cover $99 \%$ of the peak intensities in our UFS sample, $76 \%$ of the half-lengths, and most significantly, $81 \%$ of the lifetimes. It is likely that adjusting the volumetric heating higher or lower by finding a new combination of rate and duration should reproduce the remaining observed properties. 
Hansteen et al. (2014) commented that their magnetohydrodynamic modeling predicts that short low-lying loops seldom reach temperatures higher than $\log \mathrm{T}=5$, and would be difficult to observe, e.g, with the Atmospheric Imaging Assembly (AIA, Lemen et al. 2012) on the Solar Dynamics Observatory (SDO, Pesnell et al. 2012), due to insufficient spatial resolution and absorption of EUV emission in the $171 \AA$ and $193 \AA$ pass-bands. For a large range of energies the loops in our hydrodynamic model also do not heat higher than this temperature, and the simulation predicts no emission in higher temperature coronal images such as $171 \AA$ or $193 \AA$. This is because the radiative loss function peaks close to this temperature $(\log \mathrm{T} \sim 5.4)$, so a large amount of energy is required to overcome the losses. We have also independently looked at the AIA data for several cases and found no clear emission. Conversely, these pass-bands also contain transition region lines and our model does predict emission from them of $\sim 30 \mathrm{DN}_{\text {pixel }}{ }^{-1} \mathrm{~s}^{-1}$. Examining several examples, we found that the background emission in the $171 \AA$ and $193 \AA$ channels around the UFS location is at least $135 \mathrm{DN}$ and $124 \mathrm{DN}$, respectively, so any transition region emission is swamped by this background.

Note that we have not attempted to reproduce all the UFS properties simultaneously for all 108 UFS in the sample, which would be a very large undertaking. By exploring parameters in the model, however, we found that for the longest duration heating, the plasma exceeds $\log \mathrm{T}=5$ and the peak intensity in the Si IV emission no longer corresponds to the maximum loop length since that occurs at higher temperatures. These cases appear more like traditional coronal loops, but produce only very short loops $(<0.4 \mathrm{Mm})$ when they are emitting in Si IV. In fact, it is difficult to produce very long Si IV loops with extended heating: a minority $(\sim 27 \%)$ of the UFS whose lifetimes are reproduced by our simulations are longer than predicted, indicating that there is a discrepancy between the modeling and observations for the longer duration UFS that needs further investigation. For the objectives of this letter, however, we stress that the loop length is not a property that can be modified by adding more strands.

\section{SUMMARY AND DISCUSSION}

We have examined the properties of a sample of 108 UFS using IRIS Si IV SJI images, and used them to guide numerical hydrodynamic simulations. We find that in the majority of cases, the lifetimes, half-lengths, and peak intensities can be reproduced by a single thread model, suggesting that they may be spatially resolved.

Another interesting aspect of the modeling is that it seems to naturally explain why the UFS light up in segments with different brightnesses at different times, but our most significant result is that the observed UFS lifetimes can be matched with a single thread model. This has proven difficult to achieve with 'warm' coronal loops, because they are observed primarily in the cooling phase (Ugarte-Urra et al. 2009), and a model that heats them to an equilibrium at $1 \mathrm{MK}$ does not produce sufficiently high densities (Winebarger et al. 2003). This has been one of the primary drivers of the development of the multi-thread model of coronal loops. The UFS, in contrast, are observed in the heating phase, and so their lifetimes can be reproduced by bringing a single strand to a temperature of $\sim 0.5 \mathrm{MK}$ and sustaining it. Our modeling shows that densities in excess of $\log \mathrm{N}=10$ can be achieved.

We also measured the UFS widths and, if they are truly resolved, the distribution shows that they are structured on a spatial scale of $133 \mathrm{~km}$ on average. As noted earlier, a few hundred $\mathrm{km}$ seems to be a common spatial scale for many structures in the solar atmosphere and is much larger than current theoretical modeling predicts. Even if the heating mechanism itself operates on much smaller spatial scales, the plasma seems to respond to heating with coherence and collective behavior on these characteristic scales, and understanding why is becoming an important question.

We speculate that this observation tends to favor a coronal heating mechanism with some threshold as an onset condition. For example, when observing the cross-field intensity profile of a coronal loop, the atomic physics is assumed to be known, and for a single thread model the emission measure relates the loop radius to the electron density (Brooks et al. 2012). The density depends on the magnitude of the heating (Klimchuk 2006), which in turn depends in an unknown way on the released magnetic energy. In some theories of coronal heating, the amount of magnetic energy available can be related to a property that switches on above a minimum threshold. In the nanoflare reconnection concept, for example, the Poynting flux into the corona that stresses the magnetic field can be related to the mis-alignment angle between the horizontal and vertical components of the field (Klimchuk 2006). Dahlburg et al. (2005) have argued that the secondary instability occurs when this mis-alignment (shear) between the fields (current sheets) exceeds a threshold angle. In this example, our idea is that this minimum threshold angle leads to a characteristic Poynting flux, heating, density, and therefore loop radius. If the instability did not have this switch-on property, then it could presumably occur for any angle, and produce any range of energies, densities, and spatial scales.

DHB thanks Viggo Hansteen for IRIS data analysis advice. The work of DHB and HPW was performed under contract to the Naval Research Laboratory and was funded by the NASA Hinode program. This research was performed while JWR held an NRC Research Associateship award at the US Naval Research Laboratory with the support of NASA. IRIS is a NASA small explorer mission developed and operated by LMSAL with mission operations executed at NASA Ames Research center and major contributions to downlink communications funded by ESA and the Norwegian Space Centre. CHIANTI is a collaborative project involving George Mason University, the University of Michigan (USA) and the University of Cambridge (UK).

\section{REFERENCES}

Antiochos, S. K., \& Noci, G. 1986, ApJ, 301, 440

Antolin, P., \& Rouppe van der Voort, L. 2012, ApJ, 745, 152

Aschwanden, M. J., Nitta, N. V., Wuelser, J., \& Lemen, J. R. 2008, ApJ, 680,1477
Asplund, M., Grevesse, N., \& Sauval, A. J. 2005, in Astronomical Society of the Pacific Conference Series, Vol. 336, Cosmic Abundances as Records of Stellar Evolution and Nucleosynthesis, ed. T. G. Barnes, III \& F. N. Bash, 25

Athay, R. G. 1982, ApJ, 263, 982

Bradshaw, S. J., \& Cargill, P. J. 2013, ApJ, 770, 12

Bradshaw, S. J., \& Klimchuk, J. A. 2011, ApJS, 194, 26 
Bradshaw, S. J., \& Mason, H. E. 2003, A\&A, 401, 699

Brooks, D. H., Warren, H. P., \& Ugarte-Urra, I. 2012, ApJ, 755, L33

Brooks, D. H., Warren, H. P., Ugarte-Urra, I., \& Winebarger, A. R. 2013, ApJ, 772, L19

Cirtain, J. W., et al. 2013, Nature, 493, 501

Dahlburg, R. B., Klimchuk, J. A., \& Antiochos, S. K. 2005, ApJ, 622, 1191

De Pontieu, B., et al. 2011, Science, 331, 55

De Pontieu, B., et al. 2014, Sol. Phys., 289, 2733

Del Zanna, G., Dere, K. P., Young, P. R., Landi, E., \& Mason, H. E. 2015, A\&A, 582, A56

Dere, K. P., Landi, E., Mason, H. E., Monsignori Fossi, B. C., \& Young, P. R. 1997, A\&AS, 125

Feldman, U. 1983, ApJ, 275, 367

Feldman, U. 1987, ApJ, 320, 426

Feldman, U. 1998, ApJ, 507, 974
Feldman, U., Dammasch, I. E., \& Wilhelm, K. 2001, ApJ, 558, 423

Hansteen, V., et al. 2014, Science, 346, 1255757

Jing, J., Xu, Y., Cao, W., Liu, C., Gary, D., \& Wang, H. 2016, Scientific Reports, 6, 24319

Klimchuk, J. A. 2006, Sol. Phys., 234, 41

Lemen, J. R., et al. 2012, Sol. Phys., 275, 17

Okamoto, T. J., et al. 2007, Science, 318, 1577

Pereira, T. M. D., De Pontieu, B., \& Carlsson, M. 2012, ApJ, 759, 18

Pesnell, W. D., Thompson, B. J., \& Chamberlin, P. C. 2012, Sol. Phys., 275, 3

Ugarte-Urra, I., Warren, H. P., \& Brooks, D. H. 2009, ApJ, 695, 642

Warren, H. P., Ugarte-Urra, I., Doschek, G. A., Brooks, D. H., \& Williams, D. R. 2008, ApJ, 686, L131

Wikstø1, Ø., Judge, P. G., \& Hansteen, V. 1998, ApJ, 501, 895

Winebarger, A. R., Warren, H. P., \& Mariska, J. T. 2003, ApJ, 587, 439 